\title{
Anesthetic considerations for tracheobronchial surgery
}

\author{
Anna Schleicher, Harald Groeben \\ Department of Anesthesiology, Critical Care Medicine and Pain Therapy, Kliniken Essen-Mitte, Essen, Germany \\ Contributions: (I) Conception and design: None; (II) Administrative support: None; (III) Provision of study materials or patients: None; (IV) \\ Collection and assembly of data: None; (V) Data analysis and interpretation: None; (VI) Manuscript writing: All authors; (VII) Final approval of \\ manuscript: All authors. \\ Correspondence to: Harald Groeben, MD. Chairman Department of Anesthesiology, Critical Care Medicine and Pain Therapy, Kliniken Essen-Mitte, \\ Henricistr. 92, 45136 Essen, Germany. Email: h.groeben@kliniken-essen-mitte.de.
}

\begin{abstract}
Tracheobronchial pathology can be related to trauma, infection, tumor, or a combination of these. Per definition, planning for tracheobronchial surgery can be complicated by the overlap of anesthesiological interests in airway management and the primary surgical field. Therefore, following a detailed description of the stenosis, management of tracheobronchial surgery requires an interdisciplinary discussion and individualized planning of the procedure. There are several options for intraoperative ventilation depending on the exact localization of the defect. Hence, different tubes and ventilation techniques from cross-field ventilation, to jet ventilation, or even spontaneous breathing under regional anesthesia, have to be discussed. Moreover, an innovative ventilation mode called flow-controlled ventilation (FVC) has been developed, which allows to apply standard tidal volumes through a narrow-bore endotracheal tube. In addition, the Ventrain has been developed as an emergency device following the same technique of an active expiration based on the Venturi principle and a controlled gas flow. In critical situations, it allows even ventilation through the working channel of a bronchoscope. Overall, tracheobronchial surgery is performed under total intravenous anesthesia and the aim of an early extubation at the end of surgery. Airway management has to be discussed and planned between surgeon and anesthesiologist. All of the steps of the procedure need constant and clear communication.
\end{abstract}

Keywords: Anesthesia; trachea; bronchi; airway surgery

Submitted Nov 08, 2019. Accepted for publication Feb 14, 2020.

doi: $10.21037 /$ jtd.2020.02.52

View this article at: http://dx.doi.org/10.21037/jtd.2020.02.52

\section{Introduction}

The National Audit Project (NAP4) of the royal college of anaesthetists has evaluated major complications of airway management in the UK in anesthesia and intensive care and emergency medicine $(1,2)$.

This evaluation found a low rate of severe complication as brain damage or death for anesthetic airway management in general, but a high percentage of complications in surgery for acute or chronic diseases in head, neck, or trachea. Reviewers considered many of these cases to be poorly managed and planned $(1,2)$. The reviewers found significant deficits in patient assessment, anesthetic plan, and team communication.
The anesthetic management of an altered airway can be challenging in itself. This challenge can be potentiated, when this airway has to be operated upon. Therefore, tracheobronchial surgery requires meticulous interdisciplinary planning. Often the surgical technique requires an intraoperative change of the devices and sometimes the type of mechanical ventilation from conventional mechanical ventilation to jet ventilation and vice versa.

In addition, there are also requirements for the choice of general anesthesia and postoperative care. In this context comorbidities and apnea tolerance of each patient has to be taken into account. 
Overall, with all the risks involved and the requirements of each patient with the need for tracheobronchial surgery, needs individual planning following an interdisciplinary discussion of the potential options and risks.

\section{Preoperative planning}

Several reasons can lead to tracheal or bronchial stenosis. The most common reasons are airway narrowing following trauma (post-tracheostomy), tumor related, or following infection (3).

The first step for the planning of airway surgery is the exact description of the stenosis. Assessment of the smallest airway diameter, the extend of the narrowed airway distance, and the location along the trachea or bronchi. The clinical relevance can be expressed by subjective symptoms of the patients with the limitation of physical activities and the ability to speak. Often patients have to sleep in a semisitting position without the ability to lay flat. Objective diagnostics describe the anatomical stenosis with the help of MRI, CT, or ultrasound. However, all these techniques can't determine the functional disabilities, which can be measured with lung function measurements and the presence of in- and expiratory stridor. The flow-volume loops of the lung function measurements can help to assess how intra- or extrathoracic parts of the stenosis contribute to the airway pathology. An arterial blood gas analysis at rest helps to decide how the patient can compensate for the stenosis. Anatomically, the trachea consist of an intra- and an extrathoracic part. Stenosis of the intrathoracic trachea or main bronchi present with a more expiratory than inspiratory stridor, while narrowing of the extrathoracic airway leads to inspiratory stridor $(3,4)$.

As described for the mediastinal mass syndrome intrathoracic stenosis caused by airway compression by tumor or lymphatic tissue can be aggravated with the loss of sub atmospheric pressure when patients receive muscle relaxation with the induction of anesthesia (5).

An endoscopic evaluation under spontaneous breathing can help to assess the functional limitations and to evaluate the options for an anesthetic management of the airway. However, endoscopy of the airway is often not well tolerated and trauma with potential swelling and bleeding has to be carefully avoided (4).

\section{Intraoperative ventilation}

Based on the smallest diameter and distance of the stenosis, a plan for intraoperative ventilation has to be discussed between anesthesiologist and surgeon. Depending on the surgical access, ventilation has to be planned in multiple steps. For example for surgery of an intrathoracic tracheal stenosis, ventilation can start in the beginning with conventional ventilation via laryngeal mask or placement of an endotracheal tube above the stenosis. Subsequently, resection of the stenosis might require crossfield ventilation, i.e., insertion of a tube into the right or left bronchus by the surgeon. The cross-field ventilation can be accomplished with jet ventilation or insertion of a cuffed tube into one of the main bronchi. Towards the end of successful surgery, a tube has to be passed through the anatomical airway to take over the ventilation. Finally, an early endotracheal extubation of the trachea is desirable to minimize irritation and the effect of inspiratory pressure on the anastomosis $(3,4,6)$.

In general there are several potential techniques for ventilation. Small conventional tubes can be used for the initial phase of the operation in soft lesions, like tracheomalacia or mild to moderate stenosis, which provide a diameter large enough for an endotracheal tube. In some patients a laryngeal mask might be used for the first part of the operation, which offers the least trauma to the stenotic airway, but has significant limitations for the maximal pressure that can be applied.

Jet ventilation can be an alternative, but requires a free back flow to avoid high pressure beyond the stenosis and the risk of pneumothoraces. Most of the jet ventilators available have a pressure control integrated to stop ventilation in case predefined pressure limits have been reached. In these cases an alternative has to be established $(3,4,6)$.

In case the stenotic part of the trachea has to be resected, the ventilation device has to be removed temporarily. This can be achieved by keeping the device above the lesion (Jet catheter, LMA) or by switching to a cross-field ventilation below the stenosis, or by moving a small tube back and forth and relying on short periods of apnea (3). In case of resection within the proximal part of the trachea ventilation can be started with a laryngeal mask, continued with a small catheter for jet ventilation beyond the stenosis and switched back to ventilation via a laryngeal mask after completion of the anastomosis.

In a totally difficult approach, several case of tracheal surgery under spontaneous breathing and regional anesthesia (mostly thoracic epidural anesthesia) have been described (7). These cases require a patient, who can tolerated the positioning and surgical procedure 


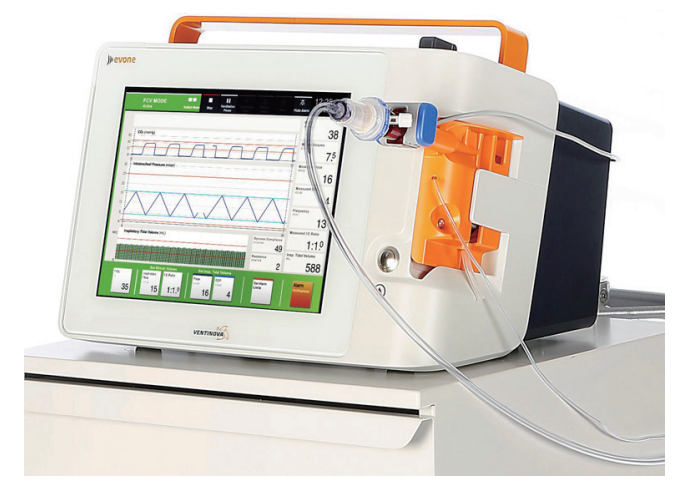

Figure 1 Flow controlled flow ventilation with the "Evone" ventilator. One main peculiarity of this technique is an active expiration according to the Venturi principle, controlled by pressure measurements distal of the cuff of the tube. This technique allows ventilation through a small catheter and leads to triangular in- and expiratory flow (lower curve on the monitor) (Ventinova Medical, Eindhoven, The Netherlands).

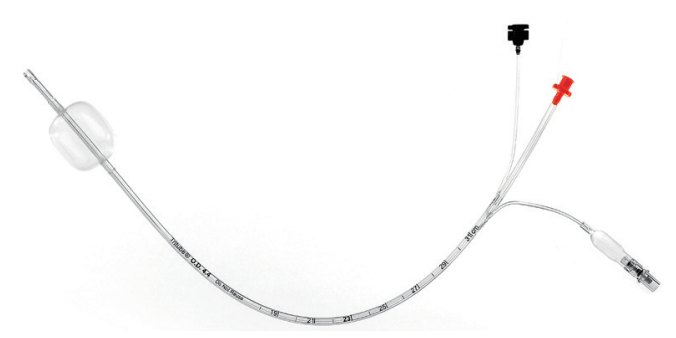

Figure 2 The Tritube is an endotracheal tube developed for flow controlled ventilation with one line for the cuff, a second line (red) for ventilation and a third line (black) for pressure measurements distal of the cuff. The tube has an inner diameter of $2.4 \mathrm{~mm}$ and an outer diameter of $4.4 \mathrm{~mm}$ (Ventinova Medical, Eindhoven, The Netherlands).

under mild sedation (for example with remifentanil and dexmedetomidine). Dexmedetomidine does not impair the respiratory drive in clinically relevant doses and has bronchodilatory qualities (7-9). However, there are only few cases reported so far and the technique is far from clinical routine (7). Alternatively, tubeless surgery can be performed under extracorporeal membrane oxygenation.

In case of laser surgery, laser-resistant endotracheal tubes have to be employed and limitation of the inspired oxygen concentration (less than $40 \%$ inspired oxygen concentration) has to be respected to avoid fire hazards. The surgical team has to take precautions with protective eyeglasses (6).

\section{Future perspective}

As a future perspective, Enk and coworkers introduced a new ventilation technique called flow-controlled ventilation (FCV, Figure 1). FCV is a new mechanical ventilation mode that maintains constant flow during inspiration and expiration with standard tidal volumes via a cuffed narrowbore endotracheal tube. The tube has an inner diameter of $2.4 \mathrm{~mm}$ and an outer diameter of $4.0 \mathrm{~mm}$ (Figure 2). One main aspect of this technique is an active expiratory control via the Venturi principle and the measurement of the airway pressure at the tip of the tube, distal of the cuff. Following the same rational, Enk et al. also invented a hand held emergency device (Ventrain, Figure 3), which enables ventilation in narrow airways through small catheters or even a working channel of a bronchoscope $(10,11)$.

\section{Anesthesia}

Besides the fact that standard jet ventilators are not suitable for the use of anesthesia gases, intermittent open airways make a continuous administration of anesthesia gases unreliable and lead to gas pollution in the operating room. Therefore, total intravenous anesthesia is the adequate choice for introduction and maintenance of general anesthesia. With respect to an early removal of the endotracheal tube remifentanil can be recommended as an opioid with a short half-life. But other opioids have been used successfully as well. Rocuronium and comparable steroid muscle relaxant drugs can be recommended to antagonize muscle relaxation with sugammadex $(3,4,6)$. The use of antisialogogues to reduce secretion is not generally recommended because secretions can be thickened and cause mucus plugging and even aggravate airway obstruction (12).

Since airway surgery requires deep anesthesia and profound muscle relaxation, EEG-monitoring (for example bispectral index monitoring) and relaxation monitoring (train-of-four monitoring) can highly be recommended $(3,4,6)$.

As the intubation and ventilation should be discussed between surgeon and anesthesiologist an extubation strategy should be discussed as well. Mostly an early extubation at the end of surgery is desirable. If a possible reintubation might be complicated by limited neck motion, an extubation via a tube exchanger tolerated with the help of local anesthesia can be advisable. In rare instances a prolonged mechanical ventilation or a chin fixation to the chest might 

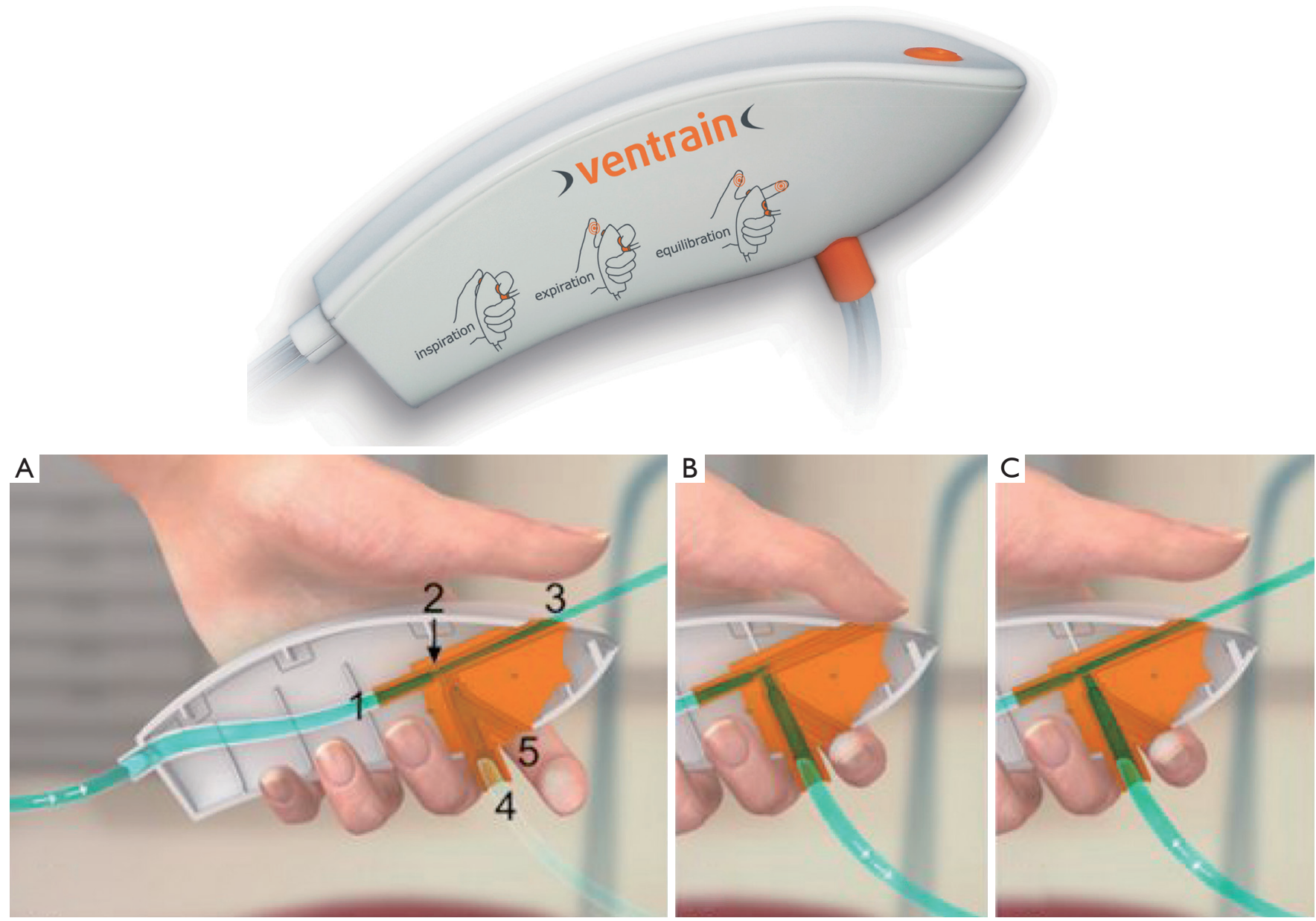

Figure 3 Ventrain is a hand-held emergency device for ventilation through small tubes, like the Tritube or in extreme situations through the work channel of a bronchoscope. The technique is based on the Venturi principle and allows an active expiration. The upper part of the figure shows the closed device with a simple description how to use the Ventrain. The lower part shows the inside of the device and the flow direction. Leaving opening 3 and 5 open results in no flow from or to the patient (A), closing opening 3 leads to flow direction to the patient (B), while closure of the opening 5 results in expiration (C).

be required to secure the surgical result.

\section{Acknowledgments}

This work was supported solely by institutional and departmental sources.

Funding: None.

\section{Footnote}

Provenance and Peer review: This article was commissioned by the Guest Editor (Servet Bölükbas) for the series "Airway Surgery", published in fournal of Thoracic Disease. This article has undergone external peer review.

Conflicts of Interest: Both authors have completed the ICMJE uniform disclosure form (available at http://dx.doi. org/10.21037/jtd.2020.02.52). The series "Airway Surgery" was commissioned by the editorial office without any funding or sponsorship. The authors have no other conflicts of interest to declare.

Ethical Statement: The authors are accountable for all aspects of the work in ensuring that questions related to the accuracy or integrity of any part of the work are appropriately investigated and resolved. 
Open Access Statement: This is an Open Access article distributed in accordance with the Creative Commons Attribution-NonCommercial-NoDerivs 4.0 International License (CC BY-NC-ND 4.0), which permits the noncommercial replication and distribution of the article with the strict proviso that no changes or edits are made and the original work is properly cited (including links to both the formal publication through the relevant DOI and the license). See: https://creativecommons.org/licenses/by-nc-nd/4.0/.

\section{References}

1. Cook TM, Woodall N, Frerk C. Major complications of airway management in the UK: results of the Fourth National Audit Project of the Royal College of Anaesthetists and the difficult airway society. Part 1: Anaesthesia. Br J Anaesth 2011;106:617-31.

2. Cook TM, MacDougall-Davis, SR. Complications and failure of airway management. Br J Anaesth 2012;109:68-85.

3. Hatipoglu Z, Turktan M, Avci A. The anesthesia of trachea and bronchus surgery. J Thorac Dis 2016;8:3442-51.

4. Pearson KL, McGuire BE. Anaesthesia for laryngotracheal surgery, including tubeless field technique. BJA Educ 2017;17:242-8.

5. Li WWL, van Boven WJP, Annema JT, et al. Management of large mediastinal masses: surgical and anesthesiological considerations. J Thorac Dis 2016;8:E175-84.

6. English J, Norris A, Bedforth N. Anaesthesia for airway surgery. BJA Educ 2006;6:28-31.

7. Okuda K, Nakanishi R. The non-intubated anesthesia for airway surgery. J Thorac Dis 2016;8:3414-9.

8. Groeben H, Mitzner W, Brown RH. Effects of the alpha2-adrenoceptor agonist dexmedetomidine on bronchoconstriction in dogs. Anesthesiology 2004;100:359-63.

9. Hoy SM, Keating GM. Dexmedetomidine: a review of its use for sedation in mechanically ventilated patients in an intensive care setting and for procedural sedation. Drugs 2011;71:1481-501.

10. Wirth S, Springer S, Spaeth, et al. Application of the novel ventilation mode flow-controlled expiration (FLEX): A crossover proof-of-principle study in lung-healthy patients. Anesth Analg 2017;125:1246-52.

11. Kristensen MS, de Wolf MWP, Rasmussen LS. Ventilation via the $2.4 \mathrm{~mm}$ internal diameter Tritube with cuff - new possibilities in airway management. Acta Anaesthesiol Scand 2017;61:580-9.

12. Mentzelopoulos SD, Tzoufi MJ. Anesthesia for tracheal and endobronchial interventions. Curr Opin Anaesthesiol 2002;15:85-94.

Cite this article as: Schleicher A, Groeben H. Anesthetic considerations for tracheobronchial surgery. J Thorac Dis 2020;12(10):6138-6142. doi: 10.21037/jtd.2020.02.52 